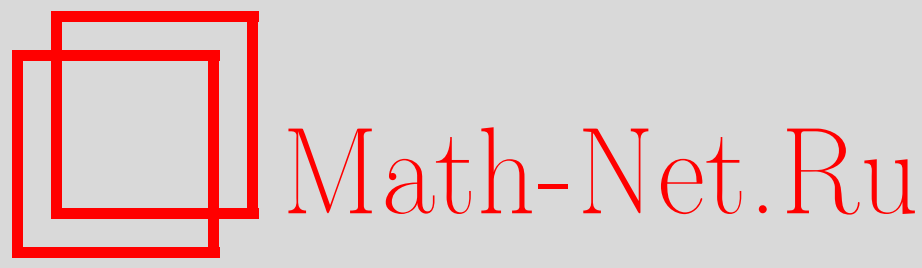

K. Р. Хуснутдинова, А. М. Самсонов, А. С. Захаров, Нелинейные длинноволновые модели для слоистых волноводов с неидеальным контактом, ТM $\Phi, 2009$, том 159, номер 3, 475-489

DOI: https://doi.org/10.4213/tmf6366

Использование Общероссийского математического портала Math-Net.Ru подразумевает, что вы прочитали и согласны с пользовательским соглашением http: //www . mathnet.ru/rus/agreement

Параметры загрузки :

IP : 34.227 .88 .159

26 апреля 2023 г., 10:19:55

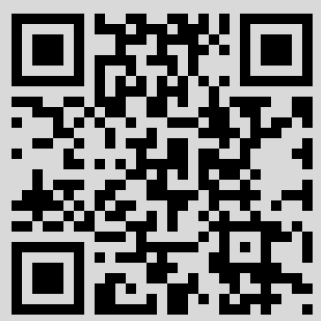




\title{
ФИЗИКА
}

Том 159, № 3

июнь, 2009

\section{НЕЛИНЕЙНЫЕ ДЛИННОВОЛНОВЫЕ МОДЕЛИ ДЛЯ СЛОИСТЫХ ВОЛНОВОДОВ С НЕИДЕАЛЬНЫМ КОНТАКТОМ}

\begin{abstract}
Предложена композитная решеточная модель для описания нелинейных волн в волноводе, склеенном из двух слоев. Сначала рассматриваются волны в ангармонической цепочке осциллирующих диполей. Показано, что соответствующая асимптотическая модель для длинных продольных волн совпадает с уравнением типа уравнения Буссинеска, ранее полученным для макроскопического волновода в рамках нелинейной теории упругости. Кроме того, показано, что в рамках данной модели нет простой аналогии между длинными продольными и длинными изгибными волнами. Для композитной решетки получены две новые модельные системы связанных уравнений типа уравнения Буссинеска для длинных нелинейных продольных волн и высказано предположение, что аналогичное описание существует в рамках динамической нелинейной теории упругости.
\end{abstract}

Ключевые слова: решеточная модель, длинная нелинейная волна, уединенная волна.

\section{1. ВВЕДЕНИЕ}

Слоистые структуры присутствуют повсюду в современной физике; особенно часто они используются в аэрокосмической промышленности и микроэлектронике. Наше исследование нелинейных волн в слоистых волноводах [1]-[3] побуждает к выводу новых модельных систем уравнений. В настоящей работе мы рассматриваем композитную решеточную модель, которая основана на сложной цепочке осциллирующих диполей [4]. Такая цепочка является естественным обобщением линейной модели, рассмотренной в работе [5] (см. также [6] и [7]), и нелинейной модели, рассмотренной в работе [8]. Введение этих моделей было мотивировано главным образом их применениями к описанию тонких пленок и твердых тел с микроструктурой. Модель, предложенная в работе [8], также была использована для описания некоторых квазиодномерных кристаллов [9]. Интерес к решеточным моделям возобновился

*Department of Mathematical Sciences, Loughborough University, Loughborough LE11 3TU, UK. E-mail: K.Khusnutdinova@lboro.ac.uk

${ }^{\dagger}$ Физико-технический институт им. А. Ф. Йоффе РАН, Санкт-Петербург 194021, Россия 
в связи с современными применениями так называемых "решеточных материалов" (см., например, статью [10]).

Хотя разностно-дифференциальные уравнения, выведенные для нашей модели, представляют значительный интерес и сами по себе, здесь мы главным образом рассматриваем случаи, когда длинноволновое поведение системы в главном порядке можно описывать относительно простыми системами связанных нелинейных дифференциальных уравнений в частных производных. Можно ли вывести такие системы как нелинейные асимптотические модели из модели композитной решетки, обладающей всеми основными степенями свободы реальных слоистых упругих структур? Это сложная задача, которая рассматривается в нашей работе. Мы отвечаем на этот вопрос с помощью вывода двух новых модельных систем связанных уравнений типа уравнения Буссинеска. На основе установленного подобия между континуальными моделями для нашей базовой решетки и макроскопическим волноводом мы высказываем предположение, что аналогичное описание существует и в рамках динамической нелинейной теории упругости. Мы заканчиваем статью обсуждением важного отличия между решениями типа уединенных волн для производных $u$ и $w$ одной из этих новых систем,

$$
\begin{aligned}
u_{t t}-u_{x x} & =u_{x} u_{x x}+u_{t t x x}-\delta(u-w), \\
w_{t t}-c^{2} w_{x x} & =\alpha w_{x} w_{x x}+\beta w_{t t x x}+\gamma(u-w),
\end{aligned}
$$

и ее редукцией к одному уравнению Буссинеска в симметричном случае.

\section{2. РЕШЕТОЧНАЯ МОДЕЛЬ ВОЛНОВОДА}

Рассмотрим связанные цепочки типа Ферми-Паста-Улама [11], состоящие из взаимодействующих частиц массы $m$ (см. рис. 1). Удобно рассматривать эту систему как ангармоническую цепочку осциллирующих диполей $\left(P_{n}, \bar{P}_{n}\right)$, каждый из которых имеет четыре степени свободы: горизонтальное $\left(u_{1}^{n}\right)$ и вертикальное $\left(u_{2}^{n}\right)$ смещения геометрического центра $O_{n}$ диполя, вращение дипольной оси в плоскости (на угол $\left.\Delta \varphi^{n}\right)$ и изменение расстояния между двумя полюсами диполя $\left(2 u_{4}^{n}\right)$. Это симметричный вариант модели, предложенной и рассмотренной в линейном приближении в связи с динамикой тонких пленок в работе [4]. Данная модель обобщает более простую цепочку диполей (с фиксированным расстоянием между полюсами), изучавшуюся как линейная модель в работе [5] (см. также [6], [7]) и как нелинейная модель в работе [8].

Смещения полюсов (см. рис. 1) можно выразить в терминах координат диполей следующим образом:

$$
\begin{aligned}
U_{1}^{n}=u_{1}^{n}-\left(l+u_{4}^{n}\right) \sin \left(\Delta \varphi^{n}\right), & U_{2}^{n}=u_{2}^{n}+\left(l+u_{4}^{n}\right) \cos \left(\Delta \varphi^{n}\right)-l, \\
\bar{U}_{1}^{n}=u_{1}^{n}+\left(l+u_{4}^{n}\right) \sin \left(\Delta \varphi^{n}\right), & \bar{U}_{2}^{n}=u_{2}^{n}-\left(l+u_{4}^{n}\right) \cos \left(\Delta \varphi^{n}\right)+l .
\end{aligned}
$$

Предполагая вращения малыми, $\Delta \varphi^{n}=u_{3}^{n} / l \ll 1$, будем использовать частичные суммы ряда Тейлора для вывода уравнений движения с точностью до квадратичных членов:

$$
U_{1}^{n}=u_{1}^{n}-u_{3}^{n}-\frac{u_{3}^{n} u_{4}^{n}}{l}+\cdots, \quad U_{2}^{n}=u_{2}^{n}+u_{4}^{n}-\frac{\left(u_{3}^{n}\right)^{2}}{2 l}+\cdots \quad \text { и т.д. }
$$



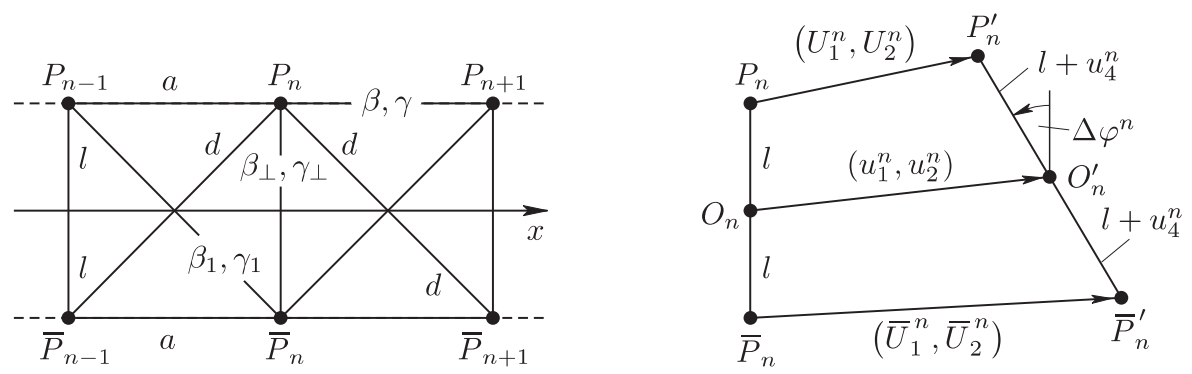

Рис. 1. Цепочка осциллирующих диполей $\left(P_{n}, \bar{P}_{n}\right)$.

Кинетическая энергия $n$-го диполя имеет вид

$$
T_{n}=\frac{M}{2}\left(\left(\dot{u}_{1}^{n}\right)^{2}+\left(\dot{u}_{2}^{n}\right)^{2}+\left(\dot{u}_{4}^{n}\right)^{2}+\left(1+\frac{u_{4}^{n}}{l}\right)^{2}\left(\dot{u}_{3}^{n}\right)^{2}\right),
$$

где $M=2 m$ - масса диполя. Потенциальная энергия $n$-го диполя определяется парными взаимодействиями между соседними частицами:

$$
\Phi_{n}=\Phi_{n, n+1}+\Phi_{\bar{n}, \overline{n+1}}+\Phi_{n, \overline{n+1}}+\Phi_{\bar{n}, n+1}+\Phi_{n-1, n}+\Phi_{\overline{n-1}, \bar{n}}+\Phi_{n-1, \bar{n}}+\Phi_{\overline{n-1}, n}+\Phi_{n, \bar{n}},
$$

где черта сверху обозначает частицу из второго ("нижнего") ряда. Пусть потенциальная энергия взаимодействия между любыми двумя соседними частицами задается как

$$
\Phi_{*}\left(\Delta r_{*}\right)=\frac{\tilde{\beta}}{2} \Delta r_{*}^{2}+\frac{\tilde{\gamma}}{3} \Delta r_{*}^{3}+\cdots,
$$

где $*$ обозначает некоторую пару индексов из $(1), \Delta r_{*}$ - изменение расстояния между соответствующими частицами, а $(\tilde{\beta}, \tilde{\gamma})$ обозначает одну из трех возможных пар констант взаимодействия, показанных на рис. 1. Изменение расстояния между одним из полюсов $n$-го диполя $\left(\widetilde{P}_{n}\right)$ и одним из полюсов $(n+1)$-го диполя $\left(\widetilde{P}_{n+1}\right)$ выражается как (см. рис. 2)

$$
\Delta r_{\widetilde{n}, \widetilde{n+1}}=\left[\left(\widetilde{U}_{1}^{n+1}-\widetilde{U}_{1}^{n}+r_{0} \cos \theta_{0}\right)^{2}+\left(\widetilde{U}_{2}^{n+1}-\widetilde{U}_{2}^{n}+r_{0} \sin \theta_{0}\right)^{2}\right]^{1 / 2}-r_{0},
$$

где $\widetilde{U}_{1}^{n}\left(\widetilde{U}_{2}^{n}\right)$ и $\widetilde{U}_{1}^{n+1}\left(\widetilde{U}_{2}^{n+1}\right)$ обозначают горизонтальные (вертикальные) смещения полюсов $\widetilde{P}_{n}$ и $\widetilde{P}_{n+1}$ соответственно. Изменение расстояния между полюсами $n$-го диполя равно $\Delta r_{n, \bar{n}}=2 u_{4}^{n}$.

Введем разности $\Delta x=\widetilde{U}_{1}^{n+1}-\widetilde{U}_{1}^{n}, \Delta y=\widetilde{U}_{2}^{n+1}-\widetilde{U}_{2}^{n}$ и предположим, что они малы по сравнению с $r_{0}: \Delta x / r_{0} \ll 1, \Delta y / r_{0} \ll 1$. Тогда можно использовать разложение

$$
\begin{aligned}
\Delta r_{\widetilde{n}, \widetilde{n+1}}= & \Delta x \cos \theta_{0}+\Delta y \sin \theta_{0}+\frac{1}{2 r_{0}}\left(\Delta x \sin \theta_{0}+\Delta y \cos \theta_{0}\right)^{2}- \\
& -\frac{1}{2 r_{0}^{2}}\left(\Delta x \cos \theta_{0}+\Delta y \sin \theta_{0}\right)\left(\Delta x \sin \theta_{0}+\Delta y \cos \theta_{0}\right)^{2}+\cdots .
\end{aligned}
$$

После этого мы выводим уравнения Эйлера-Лагранжа

$$
\frac{d}{d t}\left(\frac{\partial T_{n}}{\partial \dot{u}_{i}^{n}}\right)-\frac{\partial T_{n}}{\partial u_{i}^{n}}+\frac{\partial \Phi_{n}}{\partial u_{i}^{n}}=0
$$




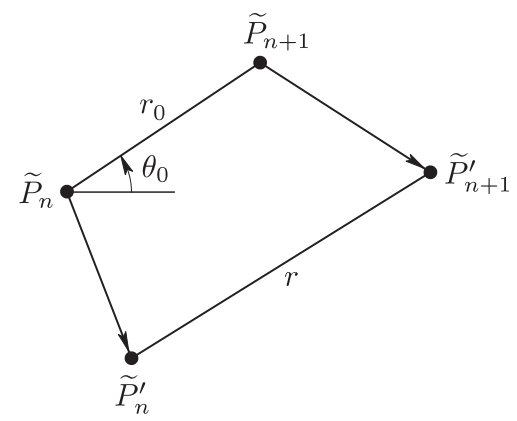

Рис. 2. Изменение расстояния между частицами $\widetilde{P}_{n}$ и $\widetilde{P}_{n+1}$.

и получаем четыре разностно-дифференциальных уравнения, записанных в компактном виде

$$
\begin{array}{r}
M \ddot{u}_{1}^{n}+\frac{\partial \Phi_{n}}{\partial u_{1}^{n}}=0, \quad M \ddot{u}_{2}^{n}+\frac{\partial \Phi_{n}}{\partial u_{2}^{n}}=0, \\
M\left[\ddot{u}_{3}^{n}\left(1+\frac{u_{4}^{n}}{l}\right)^{2}+2 \frac{\dot{u}_{3}^{n} \dot{u}_{4}^{n}}{l}\left(1+\frac{u_{4}^{n}}{l}\right)\right]+\frac{\partial \Phi_{n}}{\partial u_{3}^{n}}=0, \\
M\left[\ddot{u}_{4}^{n}-\frac{\left(\dot{u}_{3}^{n}\right)^{2}}{l}\left(1+\frac{u_{4}^{n}}{l}\right)\right]+\frac{\partial \Phi_{n}}{\partial u_{4}^{n}}=0,
\end{array}
$$

где

$$
\begin{gathered}
-\frac{\partial \Phi_{n}}{\partial u_{i}^{n}}=b_{i j} \Delta_{j}^{n}+c_{i j}\left(\Delta^{2}\right)_{j}^{n}+d_{i j} u_{j}^{n}+g_{i j k}\left(\Delta^{2}\right)_{j}^{n} \Delta_{k}^{n}+h_{i j k}\left(\left(\Delta^{2}\right)_{j}^{n}\left(\Delta^{2}\right)_{k}^{n}+\Delta_{j}^{n} \Delta_{k}^{n}\right)+ \\
+\left(l_{i j} u_{3}^{n}+\tilde{l}_{i j} u_{4}^{n}\right)\left(\Delta^{2}\right)_{j}^{n}+\left(m_{i j} u_{3}^{n}+\widetilde{m}_{i j} u_{4}^{n}\right) \Delta_{j}^{n}+p_{i j k} u_{j}^{n} u_{k}^{n}
\end{gathered}
$$

и $\Delta_{j}^{n}=u_{j}^{n+1}-u_{j}^{n-1},\left(\Delta^{2}\right)_{j}^{n}=u_{j}^{n+1}-2 u_{j}^{n}+u_{j}^{n-1}$. Здесь, как и в последующих формулах, предполагается суммирование по повторяющимся индексам.

Ненулевые коэффициенты в (5) выражаются через параметры решетки следующими явными формулами:

$$
\begin{gathered}
b_{14}=-b_{23}=\beta_{1} \sin (2 \alpha), \quad b_{i j}=-b_{j i} ; \\
c_{11}=2\left(\beta+\beta_{1} \cos ^{2} \alpha\right), \quad c_{22}=-c_{44}=2 \beta_{1} \sin ^{2} \alpha, \quad c_{33}=2\left(\beta-\beta_{1} \cos ^{2} \alpha\right) ; \\
d_{33}=-8 \beta_{1} \cos ^{2} \alpha, \quad d_{44}=-8 \beta_{1} \sin ^{2} \alpha-4 \beta_{\perp} ; \\
g_{111}=2\left(\gamma+\gamma_{1} \cos ^{3} \alpha\right)+\frac{3 \beta_{1}}{d} \cos \alpha \sin ^{2} \alpha, \\
g_{122}=g_{212}=g_{144}=2 \gamma_{1} \cos \alpha \sin ^{2} \alpha+\frac{\beta}{d \cos \alpha}+\frac{\beta_{1}}{d} \cos ^{3} \alpha-\frac{2 \beta_{1}}{d} \cos \alpha \sin ^{2} \alpha, \\
g_{133}=g_{111}-\frac{2 \beta_{1}}{d} \cos \alpha, \quad g_{234}=-g_{122}-\frac{2 \beta_{1}}{d} \cos \alpha, \\
g_{313}=-g_{111}+4 \gamma, \quad g_{324}=-g_{414}=g_{423}=g_{122}-\frac{2 \beta}{d \cos \alpha}, \quad g_{i j k}=g_{i k j} ; \\
h_{123}=-h_{114}=h_{213}=-h_{312}=h_{411}=-\gamma_{1} \sin \alpha \cos ^{2} \alpha+\frac{\beta_{1}}{d} \sin \alpha \cos ^{2} \alpha-\frac{\beta_{1}}{2 d} \sin ^{3} \alpha,
\end{gathered}
$$




$$
\begin{aligned}
& h_{224}=-h_{422}=-h_{444}=\gamma_{1} \sin ^{3} \alpha+\frac{3 \beta_{1}}{2 d} \sin \alpha \cos ^{2} \alpha, \\
& h_{334}=h_{123}+\frac{\beta}{d \sin \alpha}-\frac{\beta_{1}}{d \sin \alpha} \cos ^{2} \alpha, \\
& h_{433}=h_{411}+\frac{\beta_{1}}{d} \sin \alpha, \quad h_{i j k}=h_{i k j} ; \\
& l_{12}=-8 \gamma_{1} \sin \alpha \cos ^{2} \alpha+\frac{8 \beta_{1}}{d} \sin \alpha \cos ^{2} \alpha-\frac{4 \beta_{1}}{d} \sin ^{3} \alpha, \\
& l_{34}=l_{12}+\frac{4 \beta}{d \sin \alpha}-\frac{4 \beta_{1}}{d \sin \alpha} \cos (2 \alpha), \quad l_{i j}=l_{j i} ; \\
& \tilde{l}_{11}=-l_{12}, \quad \tilde{l}_{22}=-\tilde{l}_{44}=8 \gamma_{1} \sin ^{3} \alpha+\frac{12 \beta_{1}}{d} \sin \alpha \cos ^{2} \alpha, \\
& \tilde{l}_{33}=l_{12}+\frac{8 \beta}{d \sin \alpha}-\frac{8 \beta_{1}}{d \sin \alpha} \cos ^{2} \alpha \\
& m_{13}=8 \gamma_{1} \cos ^{3} \alpha-\frac{4 \beta_{1}}{d} \cos \alpha+\frac{12 \beta_{1}}{d} \sin ^{2} \alpha \cos \alpha, \\
& m_{24}=-8 \gamma_{1} \sin ^{2} \alpha \cos \alpha-\frac{4 \beta_{1}}{d} \cos \alpha-\frac{4 \beta_{1}}{d} \cos ^{3} \alpha+\frac{8 \beta_{1}}{d} \sin ^{2} \alpha \cos \alpha, \quad m_{i j}=-m_{j i} ; \\
& \widetilde{m}_{14}=-m_{24}-\frac{4 \beta_{1}}{d} \cos \alpha, \quad \widetilde{m}_{23}=m_{24}, \quad \widetilde{m}_{i j}=-\widetilde{m}_{j i} ; \\
& p_{334}=p_{433}=2 l_{12}-\frac{16 \beta_{1}}{d \sin \alpha} \cos ^{2} \alpha+\frac{8 \beta_{1}}{d} \sin \alpha, \\
& p_{444}=-16 \gamma_{1} \sin ^{3} \alpha-\frac{24 \beta_{1}}{d} \sin \alpha \cos ^{2} \alpha-8 \gamma_{\perp}, \quad p_{i j k}=p_{i k j} \text {. }
\end{aligned}
$$

Для проведения этих и последующих расчетов мы применяли символьные вычисления на компьютере с использованием пакета Mathematica [12] ${ }^{1)}$.

\section{3. ДЛИННОВОЛНОВЫЕ МОДЕЛИ ДЛЯ ПРОДОЛЬНЫХ И ИЗГИБНЫХ ВОЛН}

Широко распространен подход к выводу приближенных длинноволновых моделей для сложных систем на основе некоторых интуитивных физических предположений о динамике системы, использующихся для упрощения лагранжиана задачи. Однако этот подход всегда приводит к некоторому уравнению, которое может оказаться неправильной асимптотической моделью. Поэтому мы не делаем никаких априорных предположений для упрощения лагранжиана. Мы рассматриваем длинные волны, используя континуальное приближение (см., например, [6])

$$
\begin{aligned}
u_{i}^{n}(t) & =u_{i}(x, t) \\
u_{i}(x \pm a, t) & =u_{i}(x, t) \pm a u_{i_{x}}(x, t)+\frac{a^{2}}{2} u_{i_{x x}}(x, t) \pm \frac{a^{3}}{6} u_{i_{x x x}}(x, t)+\frac{a^{4}}{24} u_{i_{x x x x}}(x, t)+\cdots
\end{aligned}
$$

и асимптотический анализ возникающей сложной системы нелинейных дифференциальных уравнений в частных производных.

\footnotetext{
1)По адресу http://www-staff.lboro.ac.uk/ makk/Math-Prog_TMP.tar.gz доступны основные программы этих вычислений.
} 
Уравнения движения (4) принимают вид

$$
\begin{gathered}
M u_{1_{t t}}=2 b_{14} a\left(u_{4_{x}}+\frac{a^{2}}{6} u_{4_{x x x}}\right)+c_{11} a^{2}\left(u_{1_{x x}}+\frac{a^{2}}{12} u_{1_{x x x x}}\right)+N_{1}, \\
M u_{2_{t t}}=-2 b_{14} a\left(u_{3_{x}}+\frac{a^{2}}{6} u_{3_{x x x}}\right)+c_{22} a^{2}\left(u_{2_{x x}}+\frac{a^{2}}{12} u_{2_{x x x x}}\right)+N_{2}, \\
M\left[u_{3_{t t}}\left(1+\frac{u_{4}}{l}\right)^{2}+2 \frac{u_{3_{t}} u_{4_{t}}}{l}\left(1+\frac{u_{4}}{l}\right)\right]=2 b_{14} a\left(u_{2_{x}}+\frac{a^{2}}{6} u_{2_{x x x}}\right)+ \\
+c_{33} a^{2}\left(u_{3_{x x}}+\frac{a^{2}}{12} u_{3_{x x x x}}\right)+d_{33} u_{3}+N_{3}, \\
M\left[u_{4_{t t}}-\frac{\left(u_{3_{t}}\right)^{2}}{l}\left(1+\frac{u_{4}}{l}\right)\right]=-2 b_{14} a\left(u_{1_{x}}+\frac{a^{2}}{6} u_{1_{x x x}}\right)- \\
-c_{22} a^{2}\left(u_{4_{x x}}+\frac{a^{2}}{12} u_{4_{x x x x}}\right)+d_{44} u_{4}+N_{4},
\end{gathered}
$$

где нелинейные члены в правой части равны

$$
\begin{aligned}
N_{i}= & g_{i j k}\left(a^{2} u_{j_{x x}}+\frac{a^{4}}{12} u_{j_{x x x x}}\right)\left(2 a u_{k_{x}}+\frac{a^{3}}{3} u_{k_{x x x}}\right)+ \\
& +\left(l_{i j} u_{3}+\tilde{l}_{i j} u_{4}\right)\left(a^{2} u_{j_{x x}}+\frac{a^{4}}{12} u_{j_{x x x x}}\right)+ \\
& +h_{i j k}\left[\left(a^{2} u_{j_{x x}}+\frac{a^{4}}{12} u_{j_{x x x x}}\right)\left(a^{2} u_{k_{x x}}+\frac{a^{4}}{12} u_{k_{x x x x}}\right)+\right. \\
& \left.+\left(2 a u_{j_{x}}+\frac{a^{3}}{3} u_{j_{x x x}}\right)\left(2 a u_{k_{x}}+\frac{a^{3}}{3} u_{k_{x x x}}\right)\right]+ \\
& +\left(m_{i j} u_{3}+\widetilde{m}_{i j} u_{4}\right)\left(2 a u_{j_{x}}+\frac{a^{3}}{3} u_{j_{x x x}}\right)+p_{i j k} u_{j} u_{k}+\cdots, \quad i=1,2,3,4 .
\end{aligned}
$$

Ключевое наблюдение состоит в том, что в линейном приближении система (6) распадается на две подсистемы, одна из которых описывает взаимодействие между продольными и дипольными (или осевыми) колебаниями, а вторая - взаимодействие между поперечными и вращательными колебаниями [8]. Это позволяет вывести асимптотические длинноволновые модели для преимущественно продолъных или изгибных волн, как показано ниже. Введем безразмерные переменные

$$
\widetilde{u}_{i}=\frac{u_{i}}{A_{i}}, \quad \tilde{t}=\frac{t}{T}, \quad \tilde{x}=\frac{x}{X} .
$$

Имея в виду преимущественно продольные волны, положим $X / T=v=O(1)$ и найдем масштабирование

$$
\frac{A_{1}}{a} \sim \varepsilon^{1 / 2}, \quad \frac{A_{2}}{a} \sim \varepsilon^{3 / 2}, \quad \frac{A_{3}}{a} \sim \varepsilon^{2}, \quad \frac{A_{4}}{a} \sim \varepsilon, \quad \frac{a}{X} \sim \varepsilon^{1 / 2},
$$


при котором поведение системы в главном порядке описывается следующей подсистемой уравнений для $\widetilde{u}_{1}$ и $\widetilde{u}_{4}$ :

$$
\begin{aligned}
& M v^{2} \widetilde{u}_{1_{\tilde{t} \tilde{t}}}-a^{2}\left(2 b_{14} \widetilde{u}_{4_{\tilde{x}}}+c_{11} \widetilde{u}_{1_{\tilde{x} \tilde{x}}}\right)+ \\
&+\varepsilon a^{3}\left(2 m_{41} \widetilde{u}_{4} \widetilde{u}_{4_{\tilde{x}}}+8 h_{123} \widetilde{u}_{1_{\tilde{x}}} \widetilde{u}_{4_{\tilde{x}}}+l_{12} \widetilde{u}_{4} \widetilde{u}_{1_{\tilde{x} \tilde{x}}}-2 g_{111} \widetilde{u}_{1_{\tilde{x}}} \widetilde{u}_{1_{\tilde{x} \tilde{x}}}\right)- \\
&-\varepsilon a^{2}\left(\frac{b_{14}}{3} \widetilde{u}_{4_{\tilde{x} \tilde{x} \tilde{x}}}+\frac{c_{11}}{12} \widetilde{u}_{1_{\tilde{x} \tilde{x} \tilde{x} \tilde{x}}}\right)+O\left(\varepsilon^{2}\right)=0, \\
& 2 b_{14} \widetilde{u}_{1_{\tilde{x}}}-d_{44} \widetilde{u}_{4}+\varepsilon\left[\frac{M v^{2}}{a^{2}} \widetilde{u}_{4_{\tilde{t} \tilde{t}}}+c_{22} \widetilde{u}_{4_{\tilde{x} \tilde{x}}}+\frac{b_{14}}{3} \widetilde{u}_{1_{\tilde{x} \tilde{x} \tilde{x}}-}\right. \\
&\left.-a\left(p_{444} \widetilde{u}_{4}^{2}+2 m_{41} \widetilde{u}_{4} \widetilde{u}_{1_{\tilde{x}}}+4 h_{123}\left(\widetilde{u}_{1_{\tilde{x}}}\right)^{2}\right)\right]+O\left(\varepsilon^{2}\right)=0 .
\end{aligned}
$$

Здесь $m_{41}=-m_{14}=m_{24}+\left(4 \beta_{1} / d\right) \cos \alpha$. Из (10) можно выразить $\widetilde{u}_{4}$ в терминах $\widetilde{u}_{1}$ с помощью асимптотической реитерации:

$$
\begin{aligned}
\widetilde{u}_{4}= & \frac{2 b_{14}}{d_{44}} \widetilde{u}_{1_{\tilde{x}}}+\varepsilon\left[4 a\left(\frac{b_{14} m_{41}}{d_{44}^{2}}-\frac{h_{123}}{d_{44}}-\frac{b_{14}^{2} p_{444}}{d_{44}^{3}}\right)\left(\widetilde{u}_{1_{\tilde{x}}}\right)^{2}+\right. \\
& \left.+\frac{2 b_{14} M v^{2}}{a^{2} d_{44}^{2}} \widetilde{u}_{1_{\tilde{t} \tilde{x} \tilde{x}}}+\left(\frac{2 b_{14} c_{22}}{d_{44}^{2}}+\frac{b_{14}}{3 d_{44}}\right) \widetilde{u}_{1_{\tilde{x} \tilde{x} \tilde{x}}}\right]+O\left(\varepsilon^{2}\right) .
\end{aligned}
$$

Подставляя (11) в (9), возвращаясь к исходным размерным переменным и обозначая $u_{1}=u$, с точностью до $O\left(\varepsilon^{2}\right)$ получаем уравнение для длинных нелинейных продольных волн в виде

$$
u_{t t}-c_{0}^{2} u_{x x}=E_{1} u_{x} u_{x x}+E_{2} u_{t t x x}+E_{3} u_{x x x x}
$$

где коэффициенты $c_{0}, E_{1}, E_{2}, E_{3}$ выражаются через параметры решетки:

$$
\begin{gathered}
c_{0}^{2}=\frac{a^{2}}{M}\left(2 \beta+\beta_{\perp} \nu\right), \quad \nu=\frac{2 \beta_{1} \cos ^{2} \alpha}{\beta_{\perp}+2 \beta_{1} \sin ^{2} \alpha} \\
E_{1}=\frac{a^{2}}{M}\left[3 \beta_{\perp} \nu(\nu+1)^{2} \sin ^{2} \alpha+a\left(4 \gamma-2 \nu^{3} \gamma_{\perp} \operatorname{tg}^{3} \alpha+\frac{\gamma_{1}}{2}\left(\frac{\beta_{\perp} \nu}{\beta_{1} \cos \alpha}\right)^{3}\right)\right], \\
E_{2}=l^{2} \nu^{2}, \quad E_{3}=\frac{a^{4}}{12 M}\left(2 \beta+\beta_{\perp} \nu\left(1-3 \nu \operatorname{tg}^{2} \alpha\right)\right) .
\end{gathered}
$$

Уравнение (12) совпадает с так называемым уравнением с двумя дисперсиями, выведенным ранее для макроскопического волновода с использованием подхода нелинейной теории упругости [13] (см. также [14], [15]). Заметим, что, в частности, этот факт является аргументом (на уровне асимптотического анализа) в обсуждаемом вопросе о применимости континуальных моделей к описанию динамики тонких пленок, а также устанавливает связь между параметрами нашей решеточной модели и макроскопическими параметрами континуальной модели. Данный подход требует использования символьных вычислений; его можно применять и к другим решеточным моделям, используемым, например, в численных расчетах молекулярной динамики.

6 Теоретическая и математическая физика, т. 159, № 3, 2009 г. 
Для рассматриваемых движений приведенное выше уравнение асимптотически эквивалентно как интегрируемому уравнению нелинейной струны [16], [17]

$$
u_{t t}-c_{0}^{2} u_{x x}=E_{1} u_{x} u_{x x}+E_{23} u_{x x x x},
$$

где

$$
E_{23}=E_{2} c_{0}^{2}+E_{3}=\frac{a^{4}}{4 M}\left[2 \beta \nu \operatorname{tg}^{2} \alpha+\left(2 \beta+\beta_{\perp} \nu\right)\left(\frac{1}{3}+\nu(\nu-1) \operatorname{tg}^{2} \alpha\right)\right],
$$

так и неинтегрируемому регуляризованному уравнению Буссинеска

$$
u_{t t}-c_{0}^{2} u_{x x}=E_{1} u_{x} u_{x x}+\widetilde{E}_{23} u_{t t x x},
$$

где $\widetilde{E}_{23}=E_{23} / c_{0}^{2}$. Последнее уравнение более предпочтительно с точки зрения численного моделирования (см., например, [18], [19]).

В главном порядке соотношение между осевым смещением и горизонтальной деформацией имеет вид $u_{4}=-l \nu u_{1_{x}}+\cdots$, что можно рассматривать как решеточный аналог эффекта Пуассона в теории упругости [20], причем параметр $\nu$ соответствует коэффициенту Пуассона, тогда как члены высшего порядка в (11) дают поправки к этому соотношению (ср. [14], [15]).

Слабые (т.е. высшего порядка по $\varepsilon$ ) поперечные и вращательные движения подчинены продольным волнам и описываются следующей подсистемой уравнений на $\widetilde{u}_{2}$ и $\widetilde{u}_{3}$ :

$$
\begin{aligned}
& M v^{2} \widetilde{u}_{2_{\tilde{t} \tilde{t}}}+a^{2}\left(2 b_{14} \widetilde{u}_{3_{\tilde{x}}}-c_{22} \widetilde{u}_{2_{\tilde{x} \tilde{x}}}\right)-\varepsilon a^{3}\left(2 m_{24}\left(\widetilde{u}_{3} \widetilde{u}_{4_{\tilde{x}}}+\widetilde{u}_{3_{\tilde{x}}} \widetilde{u}_{4}\right)+8 h_{123} \widetilde{u}_{1_{\tilde{x}}} \widetilde{u}_{3_{\tilde{x}}}+\right. \\
& \left.+8 h_{224} \widetilde{u}_{2_{\tilde{x}}} \widetilde{u}_{4_{\tilde{x}}}+l_{12} \widetilde{u}_{3} \widetilde{u}_{1_{\tilde{x} \tilde{x}}}+\hat{l}_{22} \widetilde{u}_{4} \widetilde{u}_{2_{\tilde{x} \tilde{x}}}+2 g_{122}\left(\widetilde{u}_{1_{\tilde{x}}} \widetilde{u}_{2_{\tilde{x} \tilde{x}}}+\widetilde{u}_{1_{\tilde{x} \tilde{x}}} \widetilde{u}_{2_{\tilde{x}}}\right)\right)+ \\
& +\varepsilon a^{2}\left(\frac{b_{14}}{3} \widetilde{u}_{3 \tilde{x} \tilde{x} \tilde{x}}-\frac{c_{22}}{12} \widetilde{u}_{2_{\tilde{x} \tilde{x} \tilde{x} \tilde{x}}}\right)+O\left(\varepsilon^{2}\right)=0, \\
& 2 b_{14} \widetilde{u}_{2_{\tilde{x}}}+d_{33} \widetilde{u}_{3}+\varepsilon\left[-\frac{M v^{2}}{a^{2}} \widetilde{u}_{3_{\tilde{t} \tilde{t}}}+c_{33} \widetilde{u}_{3_{\tilde{x} \tilde{x}}}+\frac{b_{14}}{3} \widetilde{u}_{2_{\tilde{x} \tilde{x} \tilde{x}}}+2 a\left(p_{334} \widetilde{u}_{3} \widetilde{u}_{4}-\right.\right. \\
& \left.\left.-m_{13} \widetilde{u}_{3} \widetilde{u}_{1_{\tilde{x}}}-m_{24} \widetilde{u}_{4} \widetilde{u}_{2 \tilde{x}}-4 h_{123} \widetilde{u}_{1_{\tilde{x}}} \widetilde{u}_{2_{\tilde{x}}}\right)\right]+O\left(\varepsilon^{2}\right)=0 .
\end{aligned}
$$

Исключая $\widetilde{u}_{3}$, получаем уравнение для $\widetilde{u}_{2}$, которое в размерных переменных имеет вид

$$
u_{2 t t}-c_{0}^{2}\left(u_{2_{x}} u_{1_{x x}}+u_{2_{x x}} u_{1_{x}}\right)-l^{2} u_{2_{t t x x}}+\frac{2 a^{2} l^{2}}{M} \beta u_{2_{x x x x}}=0 .
$$

В главном порядке вращательные движения определяются из соотношения $u_{3}=$ $l u_{2_{x}}+\cdots$.

Интересно также рассмотреть случай преимущественно изгибных волн, вводя другое масштабирование:

$$
\frac{A_{1}}{a} \sim \varepsilon^{3 / 2}, \quad \frac{A_{2}}{a} \sim \varepsilon^{1 / 2}, \quad \frac{A_{3}}{a} \sim \varepsilon, \quad \frac{A_{4}}{a} \sim \varepsilon^{2}, \quad \frac{a}{X} \sim \varepsilon^{1 / 2}, \quad \frac{X}{T}=v \sim 1 .
$$

С точностью до $O\left(\varepsilon^{2}\right)$ это приводит к следующей подсистеме уравнений на $\widetilde{u}_{2}$ и $\widetilde{u}_{3}$ :

$$
\begin{aligned}
& \widetilde{u}_{2_{\tilde{t} \tilde{t}}}+\frac{a^{2}}{M v^{2}}\left[\left(2 b_{14} \widetilde{u}_{3_{\tilde{x}}}-c_{22} \widetilde{u}_{2_{\tilde{x} \tilde{x}}}\right)+\varepsilon\left(\frac{b_{14}}{3} \widetilde{u}_{3_{\tilde{x} \tilde{x} \tilde{x}}}-\frac{c_{22}}{12} \widetilde{u}_{2_{\tilde{x} \tilde{x} \tilde{x} \tilde{x}}}\right)\right]=0, \\
& \widetilde{u}_{2_{\tilde{x}}}+\frac{d_{33}}{2 b_{14}} \widetilde{u}_{3}+\frac{\varepsilon}{2 b_{14}}\left(-\frac{M v^{2}}{a^{2}} \widetilde{u}_{3_{\tilde{t} \tilde{t}}}+c_{33} \widetilde{u}_{3_{\tilde{x} \tilde{x}}}+\frac{b_{14}}{3} \widetilde{u}_{2_{\tilde{x} \tilde{x} \tilde{x}}}\right)=0 .
\end{aligned}
$$



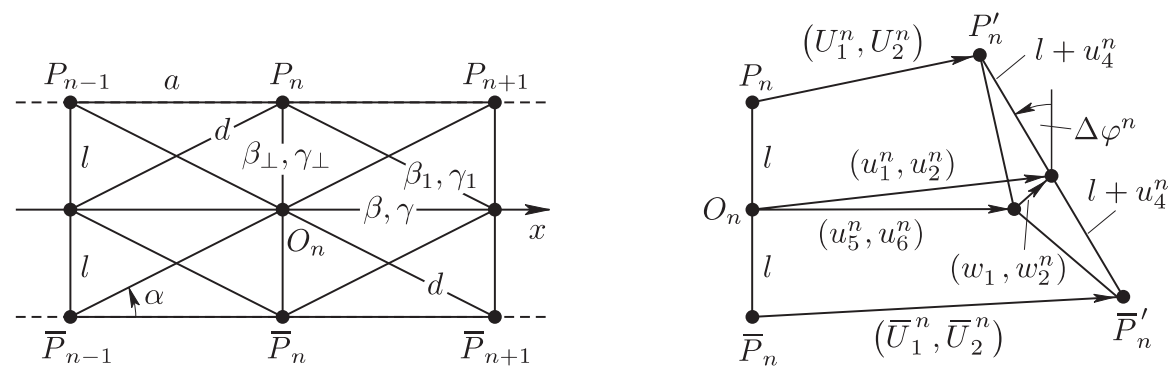

Рис. 3. Композитная решетка с идеальным контактом.

Как и выше, исключая $\widetilde{u}_{3}$ из второго уравнения с помощью асимптотической реитерации (в главном порядке $\widetilde{u}_{3}=l \widetilde{u}_{2 \tilde{x}}+\cdots$ ) и возвращаясь к исходным переменным, получаем линейное уравнение на $u_{2}$ :

$$
u_{2_{t t}}=l^{2} u_{2_{t t x x}}-\frac{2 a^{2} l^{2}}{M} \beta u_{2_{x x x x}},
$$

которое является аналогом уравнения для изгибных волн в упругих волноводах, когда учитывают эффект вращательной инерции, но пренебрегают эффектом сдвиговых деформаций (см., например, [21]).

\section{4. КОМПОЗИТНАЯ РЕШЕТКА С ИДЕАЛЬНЫМ КОНТАКТОМ}

Рассмотрим симметричную решетку, изображенную на рис. 3, для двух идентичных волноводов, спаянных друг с другом таким образом, что смещения и напряжения непрерывны при переходе через контакт (так называемый идеалъный контакт, см., например, [22]). Переменные $u_{1}^{n}, u_{2}^{n}, u_{3}^{n}, u_{4}^{n}$, соответствующие смещениям граничных частиц $P_{n}$ и $\bar{P}_{n}$, определяются, как в разделе 2 . Горизонтальные и вертикальные смещения центральных частиц $O_{n}$ описываются переменными $u_{5}^{n}$ и $u_{6}^{n}$ соответственно (см. рис. 3). Выводя дискретные и непрерывные уравнения, как и выше, и вводя безразмерные переменные (7), мы находим масштабирование для преимущественно продолъных волн в виде

$$
\begin{aligned}
& \frac{A_{1}}{a} \sim \frac{A_{5}}{a} \sim \varepsilon^{1 / 2}, \quad \frac{A_{2}}{a} \sim \frac{A_{6}}{a} \sim \varepsilon^{3 / 2}, \quad \frac{A_{3}}{a} \sim \varepsilon^{2}, \quad \frac{A_{4}}{a} \sim \varepsilon, \\
& \frac{a}{X} \sim \varepsilon^{1 / 2}, \quad \frac{X}{T}=v \sim 1 .
\end{aligned}
$$

Предполагая, что центральные частицы не сдвигаются слишком далеко от геометрического центра диполя, запишем

$$
\widetilde{u}_{1}=\widetilde{u}_{5}+\varepsilon \tilde{w}_{1}+\cdots, \quad \widetilde{u}_{2}=\widetilde{u}_{6}+\varepsilon \tilde{w}_{2}+\cdots,
$$

где $\tilde{w}_{1,2}-$ неизвестные функции. Это предположение является аналогом гипотезы о почти плоском поперечном сечении в теории упругих стержней и балок (см. монографии [14], [15]). 
Далее мы асимптотически выводим независимую асимптотическую подсистему уравнений для преимущественно продольных волн. Она следует из уравнений на $\widetilde{u}_{1}$, $\widetilde{u}_{4}$ и $\widetilde{u}_{5}$ и имеет вид

$$
\begin{aligned}
& M v^{2} \widetilde{u}_{5_{\tilde{t} \tilde{t}}}=3 a^{2}\left(\left(\beta+\beta_{1} \cos ^{2} \alpha\right) \widetilde{u}_{5 \tilde{x} \tilde{x}}-2 \beta_{1}\left(\cos ^{2} \alpha\right) \widetilde{w}_{1}\right)-\varepsilon\left(M v^{2} w_{1 \tilde{t} \tilde{t}}+\right. \\
& \left.+a^{3}\left(D_{3} \widetilde{w}_{1} \widetilde{u}_{4}+D_{4} \widetilde{w}_{1} \widetilde{u}_{5 \tilde{x}}+D_{5} \widetilde{u}_{4} \widetilde{u}_{5 \tilde{x} \tilde{x}}+D_{6} \widetilde{u}_{5_{\tilde{x}}} \widetilde{u}_{5 \tilde{x} \tilde{x}}\right)\right)+ \\
& +\varepsilon a^{2}\left(D_{7} \widetilde{w}_{1_{\tilde{x} \tilde{x}}}+D_{8} \widetilde{u}_{5_{\tilde{x} \tilde{x} \tilde{x} \tilde{x}}}\right)+O\left(\varepsilon^{2}\right), \\
& 0=\left(3 \beta_{\perp}+6 \beta_{1} \sin ^{2} \alpha\right) \widetilde{u}_{4}+3 \beta_{1} \sin (2 \alpha) \cdot \widetilde{u}_{5_{\tilde{x}}}+\varepsilon\left(\frac{M v^{2}}{a^{2}} \widetilde{u}_{4_{\tilde{t} \tilde{t}}}+\right. \\
& \left.+D_{9} \widetilde{u}_{5_{\tilde{x} \tilde{x} \tilde{x}}}+a\left(D_{10} \widetilde{u}_{4}^{2}+D_{11} \widetilde{u}_{4} \widetilde{u}_{5_{\tilde{x}}}+D_{12}\left(\widetilde{u}_{5_{\tilde{x}}}\right)^{2}\right)\right)+O\left(\varepsilon^{2}\right), \\
& M v^{2} \widetilde{u}_{5_{\tilde{t} \tilde{t}}}=3 a^{2}\left(4 \beta_{1}\left(\cos ^{2} \alpha\right) \widetilde{w}_{1}+2 \beta_{1} \sin (2 \alpha) \widetilde{u}_{4_{\tilde{x}}}+\left(\beta+2 \beta_{1} \cos ^{2} \alpha\right) \widetilde{u}_{5 \tilde{x} \tilde{x}}\right)- \\
& -\varepsilon a^{3}\left(D_{13} \widetilde{w}_{1} \widetilde{u}_{4}+D_{14} \widetilde{w}_{1} \widetilde{u}_{5_{\tilde{x}}}+D_{15} \widetilde{u}_{4} \widetilde{u}_{4_{\tilde{x}}}+D_{16} \widetilde{u}_{4_{\tilde{x}}} \widetilde{u}_{5_{\tilde{x}}}+D_{17} \widetilde{u}_{4} \widetilde{u}_{5_{\tilde{x} \tilde{x}}}+\right. \\
& \left.+D_{18} \widetilde{u}_{5_{\tilde{x}}} \widetilde{u}_{5_{\tilde{x} \tilde{x}}}\right)+\varepsilon a^{2}\left(D_{19} \widetilde{w}_{1_{\tilde{x} \tilde{x}}}+D_{20} \widetilde{u}_{4_{\tilde{x} \tilde{x} \tilde{x}}}+D_{21} \widetilde{u}_{5_{\tilde{x} \tilde{x} \tilde{x} \tilde{x}}}\right)+O\left(\varepsilon^{2}\right) \text {, }
\end{aligned}
$$

где $D_{1}, \ldots, D_{21}$ - некоторые промежуточные коэффициенты. Из уравнения (17), используя асимптотическую реитерацию, выразим $\widetilde{w}_{1}$ через $\widetilde{u}_{4}$ и $\widetilde{u}_{5}$. Тогда $\widetilde{u}_{4}$ можно найти из уравнения (18) в терминах $\widetilde{u}_{5}$, снова используя асимптотическую реитерацию. Затем из уравнения (19) выводится уравнение на $\widetilde{u}_{5}$, которое имеет следующий размерный вид:

$$
u_{5_{t t}}-c_{0}^{2} u_{5_{x x}}=F_{1} u_{5_{x}} u_{5_{x x}}+F_{2} u_{5_{t t x x}}+F_{3} u_{5_{x x x x}}+F_{4} u_{5_{t t t t}},
$$

где

$$
\begin{gathered}
c_{0}^{2}=\frac{a^{2}}{M}\left(3 \beta+2 \beta_{\perp} \nu\right), \quad \nu=\frac{2 \beta_{1} \cos ^{2} \alpha}{\beta_{\perp}+2 \beta_{1} \sin ^{2} \alpha} \\
F_{1}=\frac{a^{2}}{M}\left[6 \beta_{\perp} \nu(1+\nu)^{2} \sin ^{2} \alpha+a\left(6 \gamma-4 \nu^{3} \gamma_{\perp} \operatorname{tg}^{3} \alpha+\gamma_{1}\left(\frac{\beta_{\perp} \nu}{\beta_{1} \cos \alpha}\right)^{3}\right)\right], \\
F_{2}=\frac{2}{3}\left[l^{2} \nu^{2}-a^{2}\left(1+\frac{\beta}{\beta_{1} \cos ^{2} \alpha}\right)\right] \\
F_{3}=\frac{a^{4}}{M}\left(\frac{9}{4} \beta+\frac{2}{3} \beta_{\perp} \nu+\frac{\beta^{2}}{\beta_{1} \cos ^{2} \alpha}\right), \quad F_{4}=\frac{M}{9 \beta_{1} \cos ^{2} \alpha}
\end{gathered}
$$

и $u_{4}=-l \nu u_{5}^{\prime}+\cdots$. Заметим, что это также уравнение типа уравнения Буссинеска. Для рассматриваемых движений слабо поперечные и вращательные движения подчинены продольным волнам аналогично предыдущему разделу.

Случай преимущественно изгибных волн можно также рассмотреть, вводя масштабирование

$$
\begin{aligned}
& \frac{A_{1}}{a} \sim \frac{A_{5}}{a} \sim \varepsilon^{3 / 2}, \quad \frac{A_{2}}{a} \sim \frac{A_{6}}{a} \sim \varepsilon^{1 / 2}, \quad \frac{A_{3}}{a} \sim \varepsilon, \quad \frac{A_{4}}{a} \sim \varepsilon^{2}, \\
& \frac{a}{X} \sim \varepsilon^{1 / 2}, \quad \frac{X}{T}=v \sim 1
\end{aligned}
$$




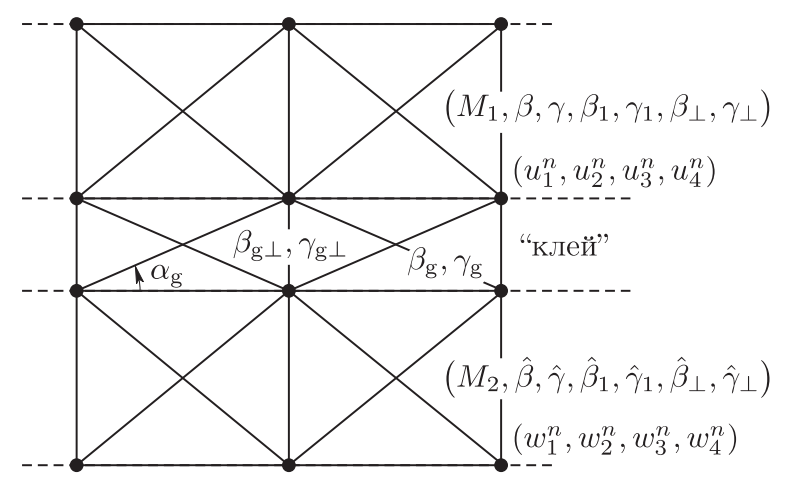

Рис. 4. Композитная решетка с неидеальным контактом.

Повторяя те же шаги, что и для продольных волн, получаем более точное уравнение для изгибных волн по сравнению со случаем однослойной решетки:

$$
u_{6_{t t}}=\frac{2}{3} l^{2}(1-\nu) u_{6_{t t x x}}-\frac{2 a^{2} l^{2}}{3 M}\left(3 \beta+\frac{3}{4} \beta_{\perp} \nu\right) u_{6_{x x x x}}+\frac{\nu M}{9 \beta_{1} \cos ^{2} \alpha} u_{6_{t t t t}} .
$$

Это аналог более точного уравнения для изгибных волн в упругом волноводе, когда учитывается отклонение, возникающее из-за сдвига (см., например, книгу [21]).

\section{5. КОМПОЗИТНАЯ РЕШЕТКА С НЕИДЕАЛЬНЫМ КОНТАКТОМ}

Для двух волноводов со слоем более мягкого материала (“клея") между ними (что обычно моделируется как неидеальный контакт, см., например, [22]) мы предлагаем композитную решеточную модель, изображенную на рис. 4; для нее мы выводим асимптотическую модель длинных нелинейных продолъных волн. Существует много исследований линейных волн в слоистых структурах с различными типами неидеального контакта (см., например, работы [22], [23] и приведенную в них библиографию), однако нам не известны какие-либо исследования, приводящие к системе связанных нелинейных волновых уравнений для слоистого волновода с неидеальным контактом. В настоящей работе мы асимптотически выводим такие системы из нашей композитной решеточной модели. Обозначения для решеточных параметров и переменных показаны на рис. 4.

Чтобы получить уравнения Эйлера-Лагранжа для композитной решетки, мы введем полную потенциальную энергию $n$-го элемента решетки:

$$
\Phi_{n}^{\text {total }}=\Phi_{n}+\widehat{\Phi}_{n}+\Phi_{n}^{\mathrm{g}}
$$

где $\Phi_{n}$ - потенциальная энергия $n$-го диполя верхнего слоя (см. формулы (1)-(3)), $\widehat{\Phi}_{n}$ выражается аналогичными формулами для потенциальной энергии $n$-го диполя нижнего слоя, а $\Phi_{n}^{g}$ - энергия взаимодействия между слоями (т.е. энергия связывающего слоя, или "клея"):

$$
\Phi_{n}^{\mathrm{g}}=\Phi_{\bar{n}, n+1}^{\mathrm{g}}+\Phi_{n, \overline{n+1}}^{\mathrm{g}}+\Phi_{n-1, n}^{\mathrm{g}}+\Phi_{n-1, \bar{n}}^{\mathrm{g}}+\Phi_{n, \bar{n}}^{\mathrm{g}} .
$$


Соответствующие формулы подобны формулам (1)-(3), но относятся к частицам, взаимодействующим через связывающий слой.

Предположим, что связывающий слой является мягким, и введем масштабирование

$$
\beta_{\mathrm{g}}=\varepsilon^{2} \tilde{\beta}_{\mathrm{g}}, \quad \beta_{\mathrm{g} \perp}=\varepsilon^{2} \tilde{\beta}_{\mathrm{g} \perp}, \quad \gamma_{\mathrm{g}}=\varepsilon^{2} \tilde{\gamma}_{\mathrm{g}}, \quad \gamma_{\mathrm{g} \perp}=\varepsilon^{2} \tilde{\gamma}_{\mathrm{g}} .
$$

Мы выводим уравнения движения, их континуальную аппроксимацию и далее используем масштабирование, найденное в разделе 2 для случая преимущественно продольных волн, для асимптотического вывода подсистемы из четырех уравнений на $\widetilde{u}_{1}, \widetilde{u}_{4}, \widetilde{w}_{1}, \widetilde{w}_{4}$, которые подобны паре связанных однослойных подсистем:

$$
\begin{aligned}
& M_{1} v^{2} \widetilde{u}_{1_{\tilde{t} \tilde{t}}}-a^{2}\left(2 b_{14} \widetilde{u}_{4_{\tilde{x}}}+c_{11} \widetilde{u}_{1_{\tilde{x} \tilde{x}}}\right)+ \\
& +\varepsilon a^{3}\left(2 m_{41} \widetilde{u}_{4} \widetilde{u}_{4_{\tilde{x}}}+8 h_{123} \widetilde{u}_{1_{\tilde{x}}} \widetilde{u}_{4_{\tilde{x}}}+l_{12} \widetilde{u}_{4} \widetilde{u}_{1_{\tilde{x} \tilde{x}}}-2 g_{111} \widetilde{u}_{1_{\tilde{x}}} \widetilde{u}_{1_{\tilde{x} \tilde{x}}}\right)- \\
& -\varepsilon a^{2}\left(\frac{b_{14}}{3} \widetilde{u}_{\tilde{x}_{\tilde{x} \tilde{x} \tilde{x}}}+\frac{c_{11}}{12} \widetilde{u}_{1 \tilde{x} \tilde{x} \tilde{x} \tilde{x}}-2 \tilde{\beta}_{\mathrm{g}}\left(\cos ^{2} \alpha_{\mathrm{g}}\right)\left(\widetilde{u}_{1}-\widetilde{w}_{1}\right)\right)+O\left(\varepsilon^{2}\right)=0 \text {, } \\
& 2 b_{14} \widetilde{u}_{1_{\tilde{x}}}-d_{44} \widetilde{u}_{4}+\varepsilon\left(\frac{M_{1} v^{2}}{a^{2}} \widetilde{u}_{4_{\tilde{t} \tilde{t}}}+c_{22} \widetilde{u}_{4_{\tilde{x} \tilde{x}}}+\frac{b_{14}}{3} \widetilde{u}_{1_{\tilde{x} \tilde{x} \tilde{x}}}-\right. \\
& \left.-a\left(p_{444} \widetilde{u}_{4}^{2}+2 m_{41} \widetilde{u}_{4} \widetilde{u}_{1_{\tilde{x}}}+4 h_{123}\left(\widetilde{u}_{1_{\tilde{x}}}\right)^{2}\right)\right)+O\left(\varepsilon^{2}\right)=0, \\
& M_{2} v^{2} \widetilde{w}_{1_{\tilde{t} \tilde{t}}}-a^{2}\left(2 \hat{b}_{14} \widetilde{w}_{4 \tilde{x}}+\hat{c}_{11} \widetilde{w}_{1_{\tilde{x} \tilde{x}}}\right)+ \\
& +\varepsilon a^{3}\left(2 \hat{m}_{41} \widetilde{w}_{4} \widetilde{w}_{4 \tilde{x}}+8 \hat{h}_{123} \widetilde{w}_{1_{\tilde{x}}} \widetilde{w}_{4 \tilde{x}}+\hat{l}_{12} \widetilde{w}_{4} \widetilde{w}_{1_{\tilde{x} \tilde{x}}}-2 \hat{g}_{111} \widetilde{w}_{1_{\tilde{x}}} \widetilde{w}_{1_{\tilde{x} \tilde{x}}}\right)- \\
& -\varepsilon a^{2}\left(\frac{\hat{b}_{14}}{3} \widetilde{w}_{4 \tilde{x} \tilde{x} \tilde{x}}+\frac{\hat{c}_{11}}{12} \widetilde{w}_{1 \tilde{x} \tilde{x} \tilde{x} \tilde{x}}-2 \tilde{\beta}_{\mathrm{g}}\left(\cos ^{2} \alpha_{\mathrm{g}}\right)\left(\widetilde{w}_{1}-\widetilde{u}_{1}\right)\right)+O\left(\varepsilon^{2}\right)=0 \text {, } \\
& 2 \hat{b}_{14} \widetilde{w}_{1_{\tilde{x}}}-\hat{d}_{44} \widetilde{w}_{4}+\varepsilon\left(\frac{M_{2} v^{2}}{a^{2}} \widetilde{w}_{4_{\tilde{t} \tilde{t}}}+\hat{c}_{22} \widetilde{w}_{4 \tilde{x} \tilde{x}}+\frac{\hat{b}_{14}}{3} \widetilde{w}_{1_{\tilde{x} \tilde{x} \tilde{x}}}-\right. \\
& \left.-a\left(\hat{p}_{444} \widetilde{w}_{4}^{2}+2 \hat{m}_{41} \widetilde{w}_{4} \widetilde{w}_{1 \tilde{x}}+4 \hat{h}_{123}\left(\widetilde{w}_{1 \tilde{x}}\right)^{2}\right)\right)+O\left(\varepsilon^{2}\right)=0 .
\end{aligned}
$$

Исключая $\widetilde{u}_{4}$ и $\widetilde{w}_{4}$ из приведенных уравнений, в главном порядке поведение системы можно описать в терминах $\widetilde{u}_{1}$ и $\widetilde{w}_{1}$ системой связанных уравнений с двумя дисперсиями, которые в исходных размерных переменных принимают вид (в обозначениях $u_{1}=u$ и $\left.w_{1}=w\right)$

$$
\begin{aligned}
u_{t t}-c_{0}^{2} u_{x x} & =E_{1} u_{x} u_{x x}+E_{2} u_{t t x x}+E_{3} u_{x x x x}-E_{4}(u-w), \\
w_{t t}-\hat{c}_{0}^{2} w_{x x} & =\widehat{E}_{1} w_{x} w_{x x}+\widehat{E}_{2} w_{t t x x}+\widehat{E}_{3} w_{x x x x}-\widehat{E}_{4}(w-u) .
\end{aligned}
$$

Коэффициенты $c_{0}, E_{1}, E_{2}, E_{3}$ определены в (13), где нужно заменить $M$ на $M_{1}$ и положить $E_{4}=\left(2 \beta_{\mathrm{g}} / M_{1}\right) \cos ^{2} \alpha_{\mathrm{g}}$. Аналогичные коэффициенты во втором уравнении получаются заменой постоянных верхнего слоя на их аналоги для нижнего слоя. Как и раньше, для рассматриваемых движений эта система асимптотически эквивалентна различным типам связанных уравнений Буссинеска. В следующем 
разделе, имея в ввиду преимущества с точки зрения численных расчетов, мы подробнее рассмотрим связанные регуляризованные уравнения Буссинеска:

$$
\begin{gathered}
u_{t t}-c_{0}^{2} u_{x x}=E_{1} u_{x} u_{x x}+\left(E_{2}+\frac{E_{3}}{c_{0}^{2}}\right) u_{t t x x}-E_{4}(u-w), \\
w_{t t}-\hat{c}_{0}^{2} w_{x x}=\widehat{E}_{1} w_{x} w_{x x}+\left(\widehat{E}_{2}+\frac{\widehat{E}_{3}}{\hat{c}_{0}^{2}}\right) w_{t t x x}-\widehat{E}_{4}(w-u) .
\end{gathered}
$$

Аналогично, ослабляя условия на коэффициенты склеивающего слоя до

$$
\beta_{\mathrm{g}}=\varepsilon \tilde{\beta}_{\mathrm{g}}, \quad \beta_{\mathrm{g} \perp}=\varepsilon \tilde{\beta}_{\mathrm{g} \perp}, \quad \gamma_{\mathrm{g}}=\varepsilon \tilde{\gamma}_{\mathrm{g}}, \quad \gamma_{\mathrm{g} \perp}=\varepsilon \tilde{\gamma}_{\mathrm{g}}
$$

и повторяя сделанные выше шаги, получаем вторую асимптотическую длинноволновую модель, которая в размерных переменных имеет вид

$$
\begin{aligned}
u_{t t}-\left(c_{0}^{2}+c_{\mathrm{g}}^{2}\right) u_{x x} & =E_{1} u_{x} u_{x x}+E_{2} u_{t t x x}+E_{3} u_{x x x x}-\left(E_{4}+E_{5} w_{x}\right)(u-w)-E_{6} w_{x x}, \\
w_{t t}-\left(\hat{c}_{0}^{2}+\hat{c}_{\mathrm{g}}^{2}\right) w_{x x} & =\widehat{E}_{1} w_{x} w_{x x}+\widehat{E}_{2} w_{t t x x}+\widehat{E}_{3} w_{x x x x}-\left(\widehat{E}_{4}+\widehat{E}_{5} u_{x}\right)(w-u)-\widehat{E}_{6} u_{x x},
\end{aligned}
$$

где коэффициенты такие же, как в (21), и, кроме того,

$$
\begin{aligned}
c_{\mathrm{g}}^{2}= & \frac{l^{2} \nu^{2}}{M_{1}}\left(\beta_{\mathrm{g} \perp}+2 \beta_{\mathrm{g}} \sin ^{2} \alpha_{\mathrm{g}}\right), \\
E_{5}=\frac{1}{M_{1}}\left[\beta_{\mathrm{g}}\left(-6 \sin ^{2} \alpha_{\mathrm{g}} \cos ^{2} \alpha_{\mathrm{g}}+(\nu+\hat{\nu})\left(3 \cos ^{2} \alpha_{1}-1\right) \operatorname{tg} \alpha \sin \alpha_{1} \cos \alpha_{1}\right)-\right. & \left.\quad-\beta_{\mathrm{g} \perp} \frac{a}{2 h}(\nu+\hat{\nu}) \operatorname{tg} \alpha-2 a \gamma_{\mathrm{g}}\left(2 \cos \alpha_{\mathrm{g}}+(\nu+\hat{\nu}) \operatorname{tg} \alpha \sin \alpha_{1}\right) \cos ^{2} \alpha_{\mathrm{g}}\right], \\
& \\
E_{6}= & \frac{a^{2}}{4 M_{1}}\left[2 \beta_{\mathrm{g}}\left(2 \cos ^{2} \alpha_{\mathrm{g}}+(\nu+\hat{\nu}) \operatorname{tg} \alpha \sin \left(2 \alpha_{\mathrm{g}}\right)+\nu \hat{\nu} \operatorname{tg}^{2} \alpha \sin ^{2} \alpha_{\mathrm{g}}\right)+\beta_{\mathrm{g} \perp} \nu \hat{\nu} \operatorname{tg}^{2} \alpha\right],
\end{aligned}
$$

а аналогичные коэффициенты во втором уравнении получаются заменой постоянных для верхнего слоя на их аналоги для нижнего слоя. На основе установленного подобия между континуальными моделями для нашей основной решеточной модели и макроскопического волновода (см. раздел 3) мы предполагаем, что подобное описание можно получить и в рамках динамической нелинейной теории упругости, используя примерно те же идеи, что и для композитной решетки.

\section{6. ЗАКЛЮЧИТЕЛЬНЫЕ ЗАМЕЧАНИЯ}

В заключение сравним численные решения связанных регуляризованных уравнений Буссинеска (22) (здесь мы запишем их в канонически масштабированном виде)

$$
\begin{aligned}
u_{t t}-u_{x x} & =u_{x} u_{x x}+u_{t t x x}-\delta(u-w), \\
w_{t t}-c^{2} w_{x x} & =\alpha w_{x} w_{x x}+\beta w_{t t x x}+\gamma(u-w)
\end{aligned}
$$

для двух наборов параметров. В симметричном случае, когда $c=\alpha=\beta=1$, уравнения (23) допускают редукцию $w=u$, где $u$ удовлетворяет регуляризованному уравнению Буссинеска

$$
u_{t t}-u_{x x}=u_{x} u_{x x}+u_{t t x x} .
$$



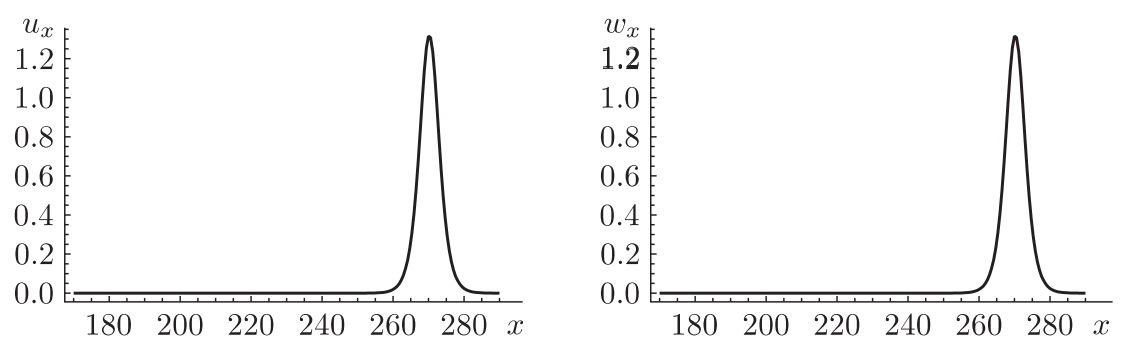

Рис. 5. Классическая уединенная волна при $t=100(c=1, \alpha=1, \beta=1$, $\gamma=\delta=0.1, v=1.2)$.
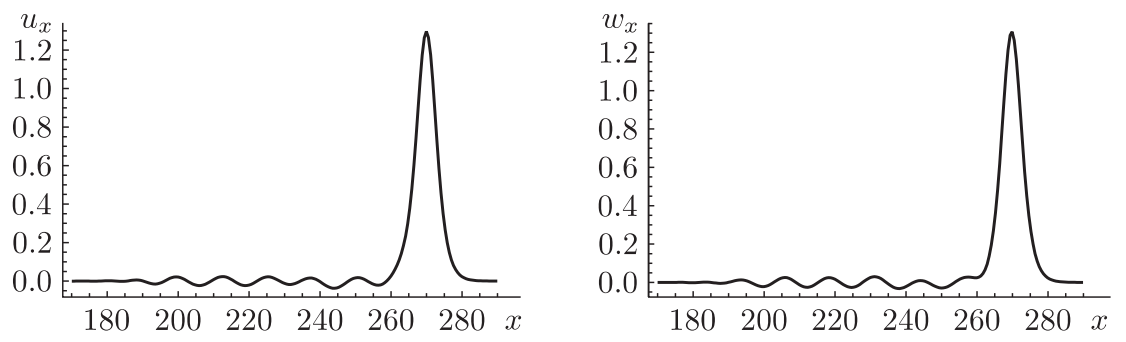

Рис. 6. Обобщенная уединенная волна при $t=100(c=1, \alpha=1, \beta=1.1$, $\gamma=\delta=0.1, v=1.2$ ).

Заметим, что данное уравнение появляется как асимптотическая модель для однородного волновода (см. раздел 2) и для симметричного двуслойного волновода с идеальной границей раздела (см. раздел 4).

Уравнение (24) имеет частные решения в виде классических (или чистых) уединенных волн для производной от $u$ :

$$
u_{x}=A \operatorname{sech}^{2}\left(\frac{x-v t}{\Lambda}\right), \quad A=3\left(v^{2}-1\right), \quad \Lambda=\frac{2 v}{\sqrt{v^{2}-1}}
$$

Численный анализ показывает, что когда симметрия нарушена, основная уединенная волна сопровождается рябью малой амплитуды, распространяющейся со скоростью уединенной волны. Таким образом, из-за резонанса между уединенной волной и распространяющейся вместе с ней периодической волной образуется обобщенная уединенная волна (см., например, работу [24] и приведенную там библиографию) вместо чистой уединенной волны. Численные решения уравнений (23) в виде классических и обобщенных уединенных волн показаны на рис. 5 и 6 . В обоих случаях начальные условия были взяты как решение (25) в виде чистой уединенной волны и для $u_{x}$, и для $w_{x}$.

Полученные результаты указывают на важное различие в поведении нелинейных волн в слоистых структурах с неидеальным контактом по сравнению с предельным случаем идеального контакта и побуждают к исследованию выведенных нами новых модельных систем, а также к получению аналогичных систем в рамках 
нелинейной теории упругости. Отмеченный эффект может быть связан с недавно наблюдавшимся отличием в скорости затухания уединенной волны деформации в таких структурах [3].

Благодарности. Данное исследование было поддержано UK EPSRC (грант № $\mathrm{EP} / \mathrm{D} 035570 / 1)$.

\section{Список литературы}

[1] K. R. Khusnutdinova, A. M. Samsonov, Phys. Rev. E, 77:6 (2008), 066603.

[2] G. V. Dreiden, K. R. Khusnutdinova, A. M. Samsonov, I. V. Semenova, J. Appl. Phys., 104:8 (2008), 086106.

[3] G. V. Dreiden, K. R. Khusnutdinova, A. M. Samsonov, I. V. Semenova, Strain, 2009.

[4] K.Р. Хуснутдинова, "Волновая динамика среды, построенной на основе двухрядной системы частиц", Глубокая переработка углеводородного сыръя, 2, ЦНИИТЭнефтехим, M., 1993, 136-145.

[5] A. Askar, Lattice Dynamical Foundations of Continuum Theories, Ser. Theoret. Appl. Mech., 2, World Sci., Singapore, 1985.

[6] G. A. Maugin, Nonlinear Waves in Elastic Crystals, Oxford Math. Monogr., Oxford Univ. Press, Oxford, 1999.

[7] Е. А. Ильюшина, K построению теории упругости неоднородных твердых тел с микроструктурой, Дис. ... канд. физ.-матем. наук, МГУ, М., 1976.

[8] К. Р. Хуснутдинова, Вестн. МГУ. Сер. 1. Матем., мех., 1992, № 2, 71-76.

[9] T. N. Dragunov, I. S. Pavlov, A. I. Potapov, Phys. Solid State, 39:1 (1997), 118-124.

[10] F. Côté, V.S. Deshpande, N. A. Fleck, A. G. Evans, Internat. J. Solids Structures, 43:20 (2006), 6220-6242.

[11] E. Fermi, J. Pasta, S. Ulam, "Studies on nonlinear problems, I", Nonlinear Wave Motion, Lecture Appl. Math., 15, ed. A. C. Newell, AMS, Providence, RI, 1974, 143-156.

[12] Mathematica, Wolfram Mathematica, http://www.wolfram.com, Wolfram Research Inc.

[13] А. М. Самсонов, Докл. АН СССР, 277:2 (1984), 332-335.

[14] A. M. Samsonov, Strain Solitons in Solids and How to Construct Them, Monogr. Surv. Pure Appl. Math., 117, CRC, Boca Raton, FL, 2001.

[15] A. V. Porubov, Amplification of Nonlinear Strain Waves in Solids, Ser. Stab. Vib. Control Syst. Ser. A, 9, World Scientific, Singapore, 2003.

[16] В. Е. Захаров, ЖЖЭТФ, 65:1 (1973), 219-225.

[17] В. Е. Захаров, А. Б. Шабат, Функи. анализ и его прил., 8:3 (1974), 43-53.

[18] T. B. Benjamin, J. L. Bona, J. J. Mahony, Philos. Trans. R. Soc. Lond. Ser. A, 272:1220 (1972), 47-78.

[19] C. I. Christov, G. A. Maugin, M. G. Velarde, Phys. Rev. E, 54:4 (1996), 3621-3638.

[20] A. E. H. Love, A Treatise on the Mathematical Theory of Elasticity, Dover, New York, 1944.

[21] E. Volterra, E. C. Zachmanoglou, Dynamics of Vibrations, Charles E. Merrill Books, Columbus, 1965.

[22] P. A. Martin, "Thin interface layers: adhesives, approximations and analysis", Elastic Waves and Ultrasonic Nondestructive Evaluation, eds. S. K. Datta, J.D. Achenbach, Y.S Rajapakse, North-Holland, Amsterdam, 1990, 217-222.

[23] O. Avila-Pozos, A. B. Movchan, J. Engrg. Math., 45:2 (2003), 155-168.

[24] C. Fochesato, F. Dias, R. Grimshaw, Phys. D, 210:1-2 (2005), 96-117. 